\title{
Deciphering the charged heavy quarkoniumlike states in chiral effective field theory
}

\author{
Bo Wang $\odot,{ }^{1,2, *}$ Lu Meng, ${ }^{1,2, \dagger}$ and Shi-Lin $\mathrm{Zhu}{ }^{1,2, *}$ \\ ${ }^{1}$ Center of High Energy Physics, Peking University, Beijing 100871, China \\ ${ }^{2}$ School of Physics and State Key Laboratory of Nuclear Physics and Technology, Peking University, \\ Beijing 100871, China
}

(Received 6 September 2020; accepted 5 November 2020; published 9 December 2020)

\begin{abstract}
We generalize the framework of chiral effective field theory to study the interactions of the isovector $D^{*} \bar{D}^{(*)}$ and $B^{*} \bar{B}^{(*)}$ systems up to the next-to-leading order, in which the long-, mid-, and short-range force contributions as well as the $S-D$ wave mixing are incorporated. Based on the Lippmann-Schwinger equation, we fit the invariant mass distributions of the elastic channels measured by the BESIII and Belle Collaborations. Our results indicate that the four charged charmoniumlike and bottomoniumlike states $Z_{c}(3900), Z_{c}(4020)$ and $Z_{b}(10610), Z_{b}(10650)$ can be well identified as the $D \bar{D}^{*}, D^{*} \bar{D}^{*}$ and $B \bar{B}^{*}, B^{*} \bar{B}^{*}$ molecular resonances. The bound state explanations are vetoed in our framework. Our study favors the $Z_{c}$ and $Z_{b}$ states are the twin partners under the heavy quark symmetry.
\end{abstract}

DOI: 10.1103/PhysRevD.102.114019

Hadrons are usually classified as the conventional quark model states ( $q \bar{q}$ mesons and $q q q$ baryons) and exotic states (glueball, hybrid, and multiquark states etc.). Hadron spectrum serves as a golden platform in investigating the low energy strong interactions. Since the discovery of $X(3872)$ in 2003 by the Belle Collaboration [1], many new states in the charmonium and bottomonium energy regions have been observed [2]. Most of these so-called $X Y Z$ states cannot be easily accommodated in the mass spectra of the quark models, which stimulated the theorists to propose various possible interpretations of these unconventional ones [3-8].

In the charmonium energy region, two charged charmoniumlike structures $Z_{c}(3900)$ and $Z_{c}(4020)$ were observed by the BESIII Collaboration in the $J / \psi \pi^{ \pm}[9]$ and $h_{c} \pi^{ \pm}[10]$ channels, respectively. The $Z_{c}(3900)$ was subsequently confirmed by the Belle [11] and Xiao et al. [12]. Later, the BESIII studied the $\left(D \bar{D}^{*}\right)^{ \pm}$and $\left(D^{*} \bar{D}^{*}\right)^{ \pm, 0}$ distributions and found the signals of $Z_{c}(3900)$ and $Z_{c}(4020)$ in the open charmed channels [13-16], respectively. The former was named as the $Z_{c}(3885)$ because the mass measured in the $\left(D \bar{D}^{*}\right)^{ \pm}$channel is about $15 \mathrm{MeV}$ smaller than that of the

\footnotetext{
*bo-wang@pku.edu.cn

†meng@pku.edu.cn

zhusl@pku.edu.cn
}

Published by the American Physical Society under the terms of the Creative Commons Attribution 4.0 International license. Further distribution of this work must maintain attribution to the author(s) and the published article's title, journal citation, and DOI. Funded by SCOAP.
$J / \psi \pi^{ \pm}$channel. Enlightened by the Ockham's razor: "Entities should not be multiplied unnecessarily," we treat the $Z_{c}(3900)$ and $Z_{c}(3885)$ as the same state that was visualized in different "microscope." After all, the mass resolution in different measurements is inequable. In the bottomonium energy region, the Belle Collaboration discovered two charged bottomoniumlike states $Z_{b}(10610)$ and $Z_{b}(10650)$ in the $\Upsilon(n S) \pi^{ \pm}(n=1,2,3)$ and $h_{b}(m P) \pi^{ \pm}$ $(m=1,2)$ invariant mass spectra [17]. Four years later, the Belle Collaboration also observed these two structures in the $B \bar{B}^{*}$ and $B^{*} \bar{B}^{*}$ channels, respectively [18].

Isospin and parity analyses indicate these $Z_{Q}^{(\prime)}(Q=c, b)$ states are the isovector particles with positive $G$ parity and negative $C$-parity ( $C$-parity for the neutral members). We will denote the $Z_{c}(3900), Z_{c}(4020)$ and $Z_{b}(10610)$, $Z_{b}(10650)$ as $Z_{c}, Z_{c}^{\prime}$ and $Z_{b}, Z_{b}^{\prime}$, respectively in the following context for simplicity. Analyses of the angular distributions favor the $J^{P}=1^{+}$assignment for the $Z_{c}$ $[13,14]$ and $Z_{b}^{(\prime)}[19]$. The $J^{P}$ quantum numbers of the $Z_{c}^{\prime}$ are undetermined yet, but the $J^{P}=1^{+}$is presumed in most works [3-8]. The minimal quark component in these $Z_{Q}^{(\prime)}$ states should be $Q \bar{Q} q \bar{q}(q=u, d)$ rather than the pure $Q \bar{Q}$ since they are the charged particles. Such a quark configuration is obviously beyond the conventional mesons and baryons, so they are dubbed the exotic hadrons. Many theoretical explanations have been proposed to understand these exotica, such as the loosely bound molecular states, compact tetraquarks, kinematical effects and so on (one can consult some comprehensive reviews [3-8] for deepgoing excavations). Besides the similarities of the decay modes, the mass differences of $\left(Z_{c}, Z_{c}^{\prime}\right)$ and $\left(Z_{b}, Z_{b}^{\prime}\right)$ almost equal to 
the mass splittings of $\left(D, D^{*}\right)$ and $\left(B, B^{*}\right)$, respectively. The large comparability between the $Z_{c}^{(\prime)}$ and $Z_{b}^{(\prime)}$ suggests that they are the partners under the heavy quark flavor symmetry. The most salient feature of the $Z_{c}, Z_{c}^{\prime}$ and $Z_{b}, Z_{b}^{\prime}$ is their proximities to the $D \bar{D}^{*}, D^{*} \bar{D}^{*}$ and $B \bar{B}^{*}, B^{*} \bar{B}^{*}$ thresholds, respectively. Therefore, the properties of the $Z_{Q}^{(\prime)}$ states are strongly related to the interactions of these open heavy flavor systems.

The $Z_{c}^{(\prime)}$ and $Z_{b}^{(\prime)}$ lie few $\mathrm{MeV}$ above the $D^{*} \bar{D}^{(*)}$ and $B^{*} \bar{B}^{(*)}$ thresholds, respectively. Thus it is natural to investigate whether the $Z_{c}^{(\prime)}$ and $Z_{b}^{(\prime)}$ are molecular resonances generated from the $D^{*} \bar{D}^{(*)}$ and $B^{*} \bar{B}^{(*)}$ interactions, respectively. In this work we exploit the chiral effective field theory $(\chi \mathrm{EFT})$ to study the $D^{*} \bar{D}^{(*)}$ and $B^{*} \bar{B}^{(*)}$ interactions up to the next-to-leading order (NLO), and then fit the experimental data to extract the resonance parameters. As the modern theory of nuclear forces [20,21], $\chi$ EFT has been extensively used to study the nucleon systems with high precision [22-27]. Within $\chi$ EFT, the effective potentials of the VP and VV systems [V and P denote the (anti)charmed/ bottom vector and pseudoscalar mesons, respectively] with the definite isospin can be respectively parametrized as

$$
\begin{gathered}
\mathcal{V}=\sum_{i=1}^{6} V_{i}\left(\boldsymbol{p}^{\prime}, \boldsymbol{p}\right) \mathcal{O}_{i}\left(\boldsymbol{p}^{\prime}, \boldsymbol{p}, \boldsymbol{\varepsilon}, \boldsymbol{\varepsilon}^{\dagger}\right), \\
\mathcal{V}^{\prime}=\sum_{i=1}^{n} V_{i}^{\prime}\left(\boldsymbol{p}^{\prime}, \boldsymbol{p}\right) \mathcal{O}_{i}^{\prime}\left(\boldsymbol{p}^{\prime}, \boldsymbol{p}, \boldsymbol{\varepsilon}, \boldsymbol{\varepsilon}^{\dagger}, \boldsymbol{\varepsilon}^{\prime}, \boldsymbol{\varepsilon}^{\prime \dagger}\right),
\end{gathered}
$$

where $\boldsymbol{p}$ and $\boldsymbol{p}^{\prime}$ denote the initial and final state momenta in the center of mass system (c.m.s.), respectively. $\boldsymbol{\varepsilon}^{(\prime)}$ and $\boldsymbol{\varepsilon}^{(\prime) \dagger}$ represent the polarization vectors of the initial and final vector mesons, respectively. $V_{i}^{(\prime)}$ are the scalar functions that can be extracted from the chiral Lagrangians, while $\mathcal{O}_{i}$ are six pertinent operators:

$$
\begin{aligned}
& \mathcal{O}_{1}=\boldsymbol{\varepsilon}^{\dagger} \cdot \boldsymbol{\varepsilon}, \quad \mathcal{O}_{2}=\left(\boldsymbol{\varepsilon}^{\dagger} \times \boldsymbol{\varepsilon}\right)(\boldsymbol{q} \times \boldsymbol{k}), \\
& \mathcal{O}_{3}=\left(\boldsymbol{q} \cdot \boldsymbol{\varepsilon}^{\dagger}\right)(\boldsymbol{q} \cdot \boldsymbol{\varepsilon}), \quad \mathcal{O}_{4}=\left(\boldsymbol{k} \cdot \boldsymbol{\varepsilon}^{\dagger}\right)(\boldsymbol{k} \cdot \boldsymbol{\varepsilon}), \\
& \mathcal{O}_{5}=\left(\boldsymbol{q} \times \boldsymbol{\varepsilon}^{\dagger}\right)(\boldsymbol{q} \times \boldsymbol{\varepsilon}), \quad \mathcal{O}_{6}=\left(\boldsymbol{k} \times \boldsymbol{\varepsilon}^{\dagger}\right)(\boldsymbol{k} \times \boldsymbol{\varepsilon}),
\end{aligned}
$$

with $\boldsymbol{q}=\boldsymbol{p}^{\prime}-\boldsymbol{p}$ the transferred momentum and $\boldsymbol{k}=\left(\boldsymbol{p}^{\prime}+\boldsymbol{p}\right) / 2$ the average momentum. For the VV system, the number of the possible operators increases drastically due to the involvement of two new polarization vectors $\boldsymbol{\varepsilon}^{\prime}$ and $\boldsymbol{\varepsilon}^{\prime \dagger}$, e.g.,

$$
\begin{aligned}
& \mathcal{O}_{1}^{\prime}=\left(\boldsymbol{\varepsilon}^{\dagger} \cdot \boldsymbol{\varepsilon}\right)\left(\boldsymbol{\varepsilon}^{\prime \dagger} \cdot \boldsymbol{\varepsilon}^{\prime}\right), \quad \mathcal{O}_{2}^{\prime}=\left(\boldsymbol{\varepsilon}^{\prime \dagger} \cdot \boldsymbol{\varepsilon}\right)\left(\boldsymbol{\varepsilon}^{\dagger} \cdot \boldsymbol{\varepsilon}^{\prime}\right), \\
& \mathcal{O}_{3}^{\prime}=\left(\boldsymbol{\varepsilon}^{\prime \dagger} \cdot \boldsymbol{\varepsilon}^{\dagger}\right)\left(\boldsymbol{\varepsilon} \cdot \boldsymbol{\varepsilon}^{\prime}\right), \quad \mathcal{O}_{4}^{\prime}=\left(\boldsymbol{q} \cdot \boldsymbol{\varepsilon}^{\prime \dagger}\right)(\boldsymbol{q} \cdot \boldsymbol{\varepsilon})\left(\boldsymbol{\varepsilon}^{\dagger} \cdot \boldsymbol{\varepsilon}^{\prime}\right), \\
& \mathcal{O}_{5}^{\prime}=\left(\boldsymbol{q} \cdot \boldsymbol{\varepsilon}^{\dagger}\right)\left(\boldsymbol{q} \cdot \boldsymbol{\varepsilon}^{\prime}\right)\left(\boldsymbol{\varepsilon}^{\prime \dagger} \cdot \boldsymbol{\varepsilon}\right), \quad \mathcal{O}_{6}^{\prime}=\left(\boldsymbol{q} \cdot \boldsymbol{\varepsilon}^{\prime \dagger}\right)\left(\boldsymbol{q} \cdot \boldsymbol{\varepsilon}^{\dagger}\right)\left(\boldsymbol{\varepsilon}^{\prime} \cdot \boldsymbol{\varepsilon}\right), \\
& \mathcal{O}_{7}^{\prime}=\left(\boldsymbol{q} \cdot \boldsymbol{\varepsilon}^{\prime}\right)(\boldsymbol{q} \cdot \boldsymbol{\varepsilon})\left(\boldsymbol{\varepsilon}^{\prime \dagger} \cdot \boldsymbol{\varepsilon}^{\dagger}\right), \ldots,
\end{aligned}
$$

where the ellipsis denotes the other possible combinations among $\boldsymbol{q}, \boldsymbol{k}, \boldsymbol{\varepsilon}^{(\prime)}$, and $\boldsymbol{\varepsilon}^{(\prime) \dagger}$ at the NLO.

Like the nuclear forces [23,24], the interactions between a pair of charmed (bottom) mesons can also be divided into the short-, mid- and long-range contributions. The $\chi \mathrm{EFT}$ does not depend on the details of the short-range dynamics $\left(r \ll 1 / m_{\pi}\right)$, which is usually mimicked by the contact interaction. Following the spirit of Eq. (1), the contact potential of the VP system is parametrized as follows,

$$
\mathcal{V}_{\mathrm{ct}}=\left(C_{0}+C_{1} \boldsymbol{q}^{2}+C_{2} \boldsymbol{k}^{2}\right) \mathcal{O}_{1}+\sum_{i=2}^{6} C_{i+1} \mathcal{O}_{i}
$$

where $C_{i}(i=0, \ldots, 7)$ are the unknown low energy constants (LECs). The $C_{0}$ and $C_{1, \ldots, 7}$ terms designate the leading order (LO) and the next-to-leading order contributions, respectively. With Eq. (2), one can construct the similar form as in Eq. (5) for the contact potential of the VV system.

The $\chi$ EFT is very good at dealing with the long- and midrange interactions, which could be calculated to any high orders theoretically. For the VP and VV systems, the longrange interaction is provided by the one-pion-exchange (OPE), which is firmly rooted in the chiral symmetry and its spontaneous breaking of quantum chromodynamics (QCD). The mid-range force arises from the two-pion-exchange (TPE). The corresponding loop diagrams are illustrated in Fig. 1. The long- and mid-range effective potentials can be obtained from the LO chiral Lagrangians,

$$
\begin{aligned}
\mathcal{L}= & i\langle\mathcal{H} v \cdot \mathcal{D} \overline{\mathcal{H}}\rangle+g\left\langle\mathcal{H} \gamma^{\mu} \gamma_{5} u_{\mu} \overline{\mathcal{H}}\right\rangle \\
& -i\langle\overline{\tilde{\mathcal{H}}} v \cdot \mathcal{D} \tilde{\mathcal{H}}\rangle+g\left\langle\overline{\tilde{\mathcal{H}}} \gamma^{\mu} \gamma_{5} u_{\mu} \tilde{\mathcal{H}}\right\rangle
\end{aligned}
$$

where $\langle\cdots\rangle$ denotes the trace in spinor space. The covariant derivative $\mathcal{D}_{\mu}=\partial_{\mu}+\Gamma_{\mu}$ and $v=(1, \mathbf{0})$ represents the fourvelocity of heavy mesons. The $\mathcal{H}$ and $\tilde{\mathcal{H}}$ denote the superfield of the charmed (bottom) mesons and anticharmed (bottom) mesons, respectively. Their expressions can be found in Refs. [28-31]. The axial coupling $g \simeq 0.57$ for the charmed mesons is extracted from the partial decay width of $D^{*+} \rightarrow D^{0} \pi^{+}$[2], while for the bottom ones average value $g \simeq 0.52$ is taken from the lattice QCD calculations $[32,33]$. The chiral connection $\Gamma_{\mu}$ and axial-vector current $u_{\mu}$ are formulated as $\Gamma_{\mu} \equiv\left[\xi^{\dagger}, \partial_{\mu} \xi\right] / 2$, and $u_{\mu} \equiv i\left\{\xi^{\dagger}, \partial_{\mu} \xi\right\} / 2$, where $\xi^{2}=U=\exp \left(i \varphi / f_{\pi}\right)$, with $\varphi$ the matrix form of the pion triplet [30], and $f_{\pi}=92.4 \mathrm{MeV}$ the pion decay constant.

Establishing the flavor wave functions of the $I^{G}\left(J^{P C}\right)=$ $1^{+}\left(1^{+-}\right) Z_{Q}^{(\prime)}$ [34] and unfolding Eq. (6) one can get the OPE potentials for the $Z_{Q}$ and $Z_{Q}^{\prime}$ states, respectively,

$$
\mathcal{V}_{\mathrm{OPE}}=-\frac{g^{2}}{4 f_{\pi}^{2}} \frac{\mathcal{O}_{3}}{\boldsymbol{q}^{2}+m_{\pi}^{2}},
$$




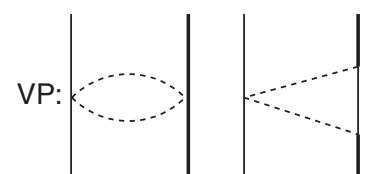

(a)

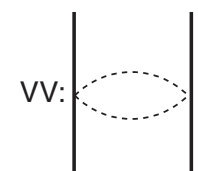

(a)

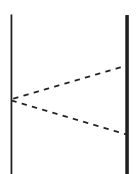

(c)

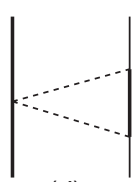

(d)

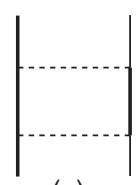

(e)

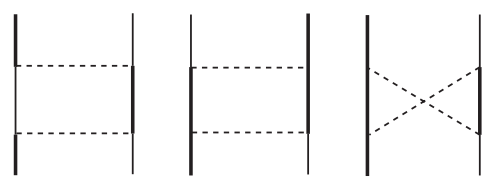

(f)

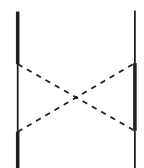

(i)

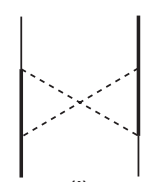

(j)

FIG. 1. The two-pion-exchange contributions to the VP and VV interactions, where we use the thick, thin, and dashed lines to denote the charmed (bottom) vector, pesudoscalar mesons and pion, respectively.

$\mathcal{V}_{\mathrm{OPE}}^{\prime}=-\frac{g^{2}}{4 f_{\pi}^{2}} \frac{\left(\mathcal{O}_{3}^{\prime}-\mathcal{O}_{2}^{\prime}\right) \boldsymbol{q}^{2}+\mathcal{O}_{4}^{\prime}+\mathcal{O}_{5}^{\prime}-\mathcal{O}_{6}^{\prime}-\mathcal{O}_{7}^{\prime}}{\boldsymbol{q}^{2}+m_{\pi}^{2}}$

with $m_{\pi}$ the pion mass, and $\boldsymbol{q}^{2}=p^{2}+p^{\prime 2}-2 p p^{\prime} \cos \vartheta$ (where $p=|\boldsymbol{p}|, p^{\prime}=\left|\boldsymbol{p}^{\prime}\right|$, and $\vartheta$ is the scattering angle in the c.m.s. of VP and VV). In the Breit approximation [35], the effective potential $\mathcal{V}$ from the scattering amplitude $\mathcal{M}$ reads $\mathcal{V}=-\mathcal{M} / \sqrt{\Pi_{i} 2 m_{i} \Pi_{f} 2 m_{f}}\left(m_{i}\right.$ and $m_{f}$ stand for the masses of initial and final states, respectively.).

Similarly, the midrange potential provided by the loop diagrams in Fig. 1 can be calculated with the one-pion and two-pion coupling vertices in Eq. (6) (for the calculation details one can consult Refs. [36,37]). In heavy quark limit, the two-particle-irreducible TPE potential can be formulated via a concise form,

$$
\mathcal{V}_{\mathrm{TPE}}^{(\prime)}=V_{1}^{(\prime)} \mathcal{O}_{1}^{(\prime)}
$$

with

$$
\begin{aligned}
V_{1}^{\prime}= & V_{1}=-\frac{24\left(4 g^{2}+1\right) m_{\pi}^{2}+\left(38 g^{2}+5\right) \boldsymbol{q}^{2}}{2304 \pi^{2} f_{\pi}^{4}} \\
& +\frac{6\left(6 g^{2}+1\right) m_{\pi}^{2}+\left(10 g^{2}+1\right) \boldsymbol{q}^{2}}{768 \pi^{2} f_{\pi}^{4}} \ln \frac{m_{\pi}^{2}}{\left(4 \pi f_{\pi}\right)^{2}} \\
& +\frac{4\left(4 g^{2}+1\right) m_{\pi}^{2}+\left(10 g^{2}+1\right) \boldsymbol{q}^{2}}{384 \pi^{2} f_{\pi}^{4} y} \varpi \arctan \frac{y}{\varpi},
\end{aligned}
$$

where $\varpi=\sqrt{\boldsymbol{q}^{2}+4 m_{\pi}^{2}}$, and $y=\sqrt{2 p p^{\prime} \cos \vartheta-p^{2}-p^{\prime 2}}$.

The $Z_{Q}$ and $Z_{Q}^{\prime}$ are observed in the $e^{+} e^{-} \rightarrow \pi \mathrm{VP}$ and $e^{+} e^{-} \rightarrow \pi \mathrm{VV}$ processes, respectively. So we simulate the two transitions and fit the invariant mass spectra of the VP and VV pair. The reaction is illustrated in Fig. 2, where graphs 2(a) and 2(b) describe the continuum and resonance contributions, respectively. In Fig. 2(b) we need to cope with the $\operatorname{VP}(V)$ rescatterings, since they account for the dynamical generation of the $Z_{Q}^{(\prime)}$. Additionally, we also need to mimic the $\gamma^{*} \rightarrow \pi \mathrm{VP}(\mathrm{V})$ coupling, which can be depicted by the following effective Lagrangians

$$
\mathcal{L}_{\gamma^{*} \pi \mathrm{VP}(\mathrm{V})}=g_{\gamma} \mathcal{F}^{\mu \nu} \mathcal{P}_{\mu \nu}+g_{\gamma}^{\prime} \epsilon^{\alpha \beta \mu \nu} \mathcal{F}_{\alpha \beta} \mathcal{P}_{\mu \nu}^{\prime} v^{\lambda} u_{\lambda},
$$

where $g_{\gamma}^{(\prime)}$ designate the effective coupling constants, and $\mathcal{F}^{\mu \nu}$ is the field strength tensor of the virtual photon. $\mathcal{P}_{\mu \nu}^{(\prime)}$ are the antisymmetric tensors that constructed as $\mathcal{P}_{\mu \nu}=\left(\tilde{P}_{\mu}^{\dagger} u_{\nu} P^{\dagger}-\tilde{P}_{\nu}^{\dagger} u_{\mu} P^{\dagger}\right)-\left(\tilde{P}^{\dagger} u_{\mu} P_{\nu}^{\dagger}-\tilde{P}^{\dagger} u_{\nu} P_{\mu}^{\dagger}\right)$ and $\mathcal{P}_{\mu \nu}^{\prime}=$ $\tilde{P}_{\mu}^{\dagger} P_{\nu}^{\dagger}-\tilde{P}_{\nu}^{\dagger} P_{\mu}^{\dagger}$, where $\left(\tilde{P}_{\mu} / \tilde{P}\right) P_{\mu} / P$ denote the (anti) charmed (bottom) vector/pseudoscalar meson fields (e.g., see Refs. [30,31]), and $u_{\mu}$ is the axial-vector field.

Equipped with the above effective potentials, the VP and VV production amplitudes $\mathcal{U}(E, \boldsymbol{p})$ can be obtained by solving the following Lippmann-Schwinger equation (LSE),

$$
\begin{aligned}
\mathcal{U}(E, \boldsymbol{p})= & \mathcal{M}(E, \boldsymbol{p}) \\
& +\int \frac{d^{3} \boldsymbol{q}}{(2 \pi)^{3}} \mathcal{V}(E, \boldsymbol{p}, \boldsymbol{q}) \mathcal{G}(E, \boldsymbol{q}) \mathcal{U}(E, \boldsymbol{q}),
\end{aligned}
$$

where $\mathcal{M}(E, \boldsymbol{p})$ denotes the production vertex from Eq. (11) and $E$ is the invariant mass of the paired $\operatorname{VP}(\mathrm{V})$. The Green's function $\mathcal{G}(E, \boldsymbol{q})$ is given as

$\mathcal{G}(E, \boldsymbol{q})=\frac{2 \mu}{\boldsymbol{p}^{2}-\boldsymbol{q}^{2}+i \epsilon}, \quad|\boldsymbol{p}|=\sqrt{2 \mu\left(E-m_{\mathrm{th}}\right)}$,

with $\mu$ and $m_{\text {th }}$ the reduced mass and threshold of the $\mathrm{VP}(\mathrm{V})$ systems, respectively. The potentials in Eqs. (5) and

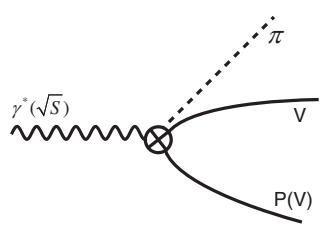

(a)

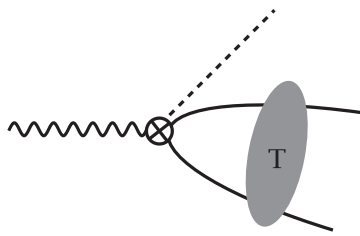

(b)
FIG. 2. Graphs (a) and (b) represent the continuum and signal channel contributions, respectively. The wiggly line denotes the virtual photon, and other notations are the same as those in Fig. 1. The gray blob in graph (b) signifies the rescatterings of VP and VV. 
(7)-(10) are given in the plane wave helicity state basis in the c.m.s. of the $\operatorname{VP}(\mathrm{V})$ systems, whereas the physical observables are usually defined in terms of partial waves, i.e., the $|\ell s j\rangle$ basis [where $\ell, s$ and $j$ represent the orbital angular momentum, total spin and total angular momentum of the $\operatorname{VP}(\mathrm{V})$ systems, respectively]. So it is desirable to obtain the above effective potentials in the partial wave decomposition. This can be easily done via [38]

$$
\begin{aligned}
\mathcal{V}_{\ell, \ell^{\prime}}= & \int d \hat{\boldsymbol{p}}^{\prime} \int d \hat{\boldsymbol{p}} \sum_{m_{\ell^{\prime}}=-\ell^{\prime}}^{\ell^{\prime}}\left\langle\ell^{\prime}, m_{\ell^{\prime}} ; s, m_{j}-m_{\ell^{\prime}} \mid j, m_{j}\right\rangle \\
& \times \sum_{m_{\ell}=-\ell}^{\ell}\left\langle\ell, m_{\ell} ; s, m_{j}-m_{\ell} \mid j, m_{j}\right\rangle \mathcal{Y}_{\ell^{\prime} m_{\ell^{\prime}}}^{*}\left(\theta^{\prime}, \phi^{\prime}\right) \\
& \times \mathcal{Y}_{\ell m_{\ell}}(\theta, \phi)\left\langle s, m_{j}-m_{\ell^{\prime}}|\mathcal{V}| s, m_{j}-m_{\ell}\right\rangle,
\end{aligned}
$$

with $\mathcal{Y}_{\ell m_{\ell}}$ the spherical harmonics. The remaining matrix element $\left\langle s, m_{j}-m_{\ell^{\prime}}|\mathcal{V}| s, m_{j}-m_{\ell}\right\rangle$ in spin space can be directly calculated with the coupled spin multiplets $\left|1, m_{s}\right\rangle$, which are the products of one-body spin states.

As demonstrated in the nucleon systems, the $S$ - and $D$-wave mixing effect plays an important role [22-25]. This effect can be easily taken into account in the LSE framework, in which the effective potential becomes a $2 \times 2$ matrix. After performing the partial wave decomposition via Eq. (14), the contact potential that incorporates the $S-D$ mixing reads,

$$
\left[\mathcal{V}_{\mathrm{ct}}\right]_{\ell, \ell^{\prime}}=\left[\begin{array}{cc}
\tilde{C}_{\mathrm{s}}+C_{\mathrm{s}}\left(p^{2}+p^{\prime 2}\right) & C_{\mathrm{sd}} p^{2} \\
C_{\mathrm{sd}} p^{\prime 2} & 0
\end{array}\right],
$$

where $\tilde{C}_{\mathrm{s}}, C_{\mathrm{s}}$, and $C_{\mathrm{sd}}$ are the so-called partial wave LECs. Their values will be fixed by fitting the experimental data.

Iteration of the potential $\mathcal{V}_{\ell, \ell^{\prime}}$ in the LSE requires suppressing the high momenta contribution to avoid divergence, since the $\chi \mathrm{EFT}$ is only valid in low momenta region $q \ll \Lambda_{\chi} \approx 1 \mathrm{GeV}$. The Gaussian regulator is commonly used [24,27,39], i.e., $\mathcal{V}_{\ell, \ell^{\prime}} \rightarrow \mathcal{V}_{\ell, \ell^{\prime}} \exp \left(-p^{\prime 2} / \Lambda^{2}-p^{2} / \Lambda^{2}\right)$, where $\Lambda$ is the cutoff parameter. For the nucleon-nucleon scattering when the high order corrections are included [24,27], the cutoff parameter $\Lambda$ is normally chosen to be around $0.5 \mathrm{GeV}$. We leave it as a free parameter and determine its value by fitting the experimental line shapes.

In terms of the production amplitude in Eq. (12), the differential decay width for $\gamma^{*} \rightarrow \pi \mathrm{VP}(\mathrm{V})$ reads

$$
\frac{d \Gamma}{d E}=\frac{1}{12(\sqrt{s})^{2}(2 \pi)^{3}}|\mathcal{U}(E)|^{2}\left|\boldsymbol{k}_{1}\right|\left|\boldsymbol{k}_{2}^{*}\right|,
$$

where $\sqrt{s}$ is the center-of-mass energy of the $e^{+} e^{-}$ collision. $\boldsymbol{k}_{1}$ and $\boldsymbol{k}_{2}^{*}$ are the three momentum of the spectator $\pi$ in the c.m.s. of $e^{+} e^{-}$and the three momentum of $\mathrm{P}(\mathrm{V})$ in the c.m.s. of $\mathrm{VP}(\mathrm{V})$, respectively.
We essentially have four free parameters [three partial wave LECs in Eq. (15) and a cutoff $\Lambda$ ] to fit the experimental lineshapes. For the $Z_{c}^{(\prime)}$ and $Z_{b}^{(\prime)}$ states, we try to fit the $D^{*} \bar{D}^{(*)}$ and $B^{*} \bar{B}^{(*)}$ invariant mass distributions measured by the BESIII [14,16] and Belle [18] Collaborations, respectively. The fitted line shapes and parameters are given in Fig. 3 and Table I, respectively. We find the experimental data can be fitted quantitatively well with the potentials up to the NLO in our approach. Four sharp peaks appear around $3.88,4.02,10.61$, and $10.65 \mathrm{GeV}$ for each distribution, which correspond to the $Z_{c}(3900), Z_{c}(4020), Z_{b}(10610)$, and $Z_{b}(10650)$ signals in experiments, respectively. With the fitted parameters in Table I as inputs, we search for the poles of the $T$ matrix in the second (unphysical) Riemann sheet, which can be achieved through analytical continuation of the Green's function $\mathcal{G}(p+i \epsilon)$ in Eq. (13),

$$
\mathcal{G}^{b}(p+i \epsilon) \equiv \mathcal{G}^{a}(p+i \epsilon)-2 i \operatorname{Im} \mathcal{G}^{a}(p+i \epsilon),
$$

where $\mathcal{G}^{a}$ and $\mathcal{G}^{b}$ denote the Green's function defined in the first (physical) and second Riemann sheet, respectively.

We find a pole for each system in the second Riemann sheet with the pole positions given in Table I. In other words, the $D^{*} \bar{D}^{(*)}$ and $B^{*} \bar{B}^{(*)}$ interactions generate the molecular resonances $Z_{c}^{(\prime)}$ and $Z_{b}^{(\prime)}$. This can be qualitatively understood. When the $\gamma^{*}$ "emits" a pion, the residual phase spaces for the $\operatorname{VP}(\mathrm{V})$ systems are small. Thus once the $\operatorname{VP}(\mathrm{V})$ are created near their thresholds, they move slowly and have enough time to interact with each other. If the interaction is attractive enough, a bound state is formed, which could not decay into its component mesons. If the interaction is not attractive enough but has a barrier to confine the two mesons for a finite time, a molecular resonance with a certain lifetime is produced.

Our extracted masses are all consistent with the experimental measurements $[14,16,18]$, but the widths in our study are smaller than those of the experimental data. We do not consider the inelastic channel $J / \psi \pi[\Upsilon(n S) \pi]$ and $h_{c} \pi\left[h_{b}(m P) \pi\right]$ contributions (see Refs. [40-43] for a couple-channel approach). These inelastic channels would contribute additional partial decay widths. These inelastic processes occur at very short distance and cannot be accommodated within the $\chi$ EFT framework. On the other hand, the coupling strength between $Z_{Q}^{(\prime)}$ and the inelastic channels is not strong, since the experimental measurements indicate that the elastic channels dominate the decay widths of $Z_{c}$ [13] and $Z_{b}^{(\prime)}$ [18]. Therefore, the corrections from the inelastic channels to the widths of $Z_{Q}^{(\prime)}$ shall not be significant. From Fig. 3, the signal line shapes deviate from the moderate Breit-Wigner distribution, which are dramatically distorted by the strong coupling of $\operatorname{VP}(\mathrm{V})$. The classical Breit-Wigner function is not good enough to describe these typical very-near-threshold states. 

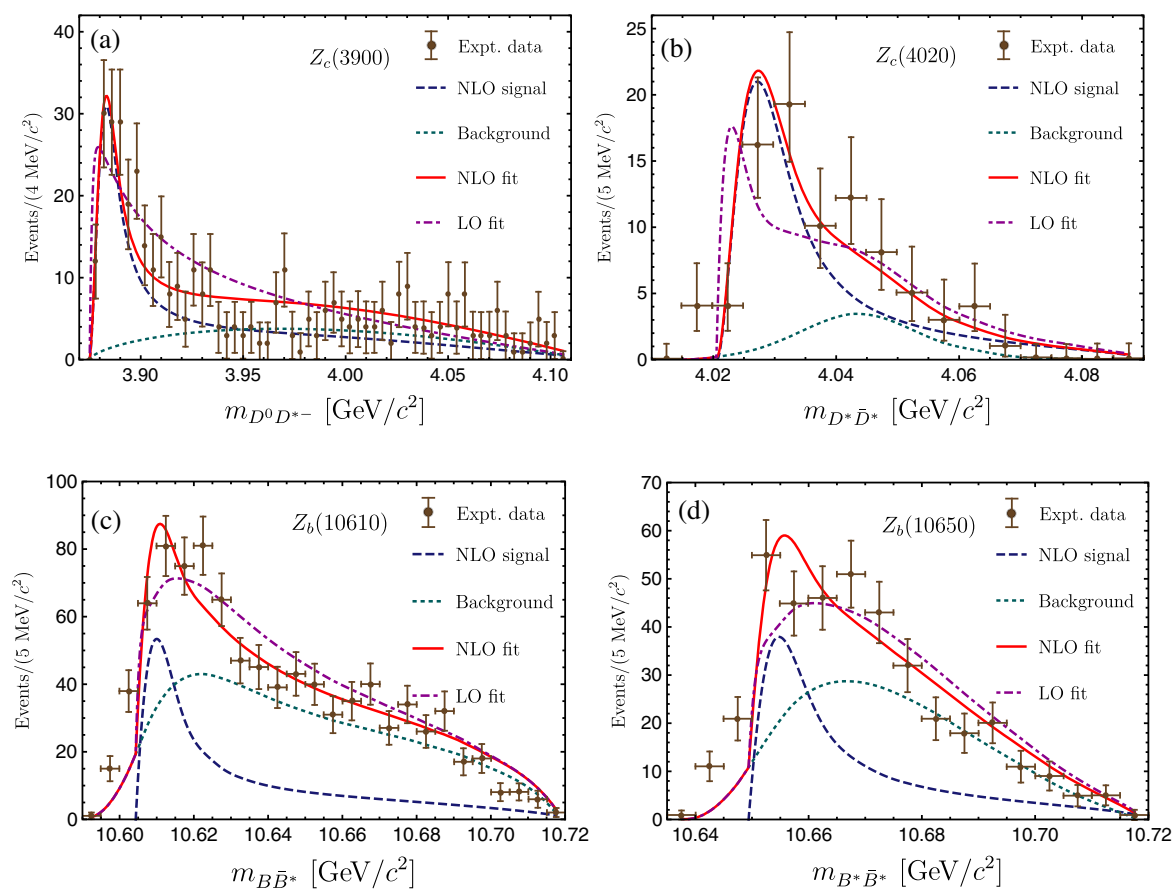

FIG. 3. The $D^{*} \bar{D}^{(*)}$ and $B^{*} \bar{B}^{(*)}$ invariant mass distributions in $e^{+} e^{-} \rightarrow \pi \mathrm{VP}(\mathrm{V})$ transitions. The data with error bars in (a), (b), and (c)/ (d) are taken from Refs. [14,16,18] at $\sqrt{s}=4.26,4.23$, and $10.86 \mathrm{GeV}$, respectively. The red solid, dark-blue dashed, purple dot-dashed, and dark-cyan dotted lines denote the NLO fit, NLO signal, LO fit, and background contributions (extracted from the corresponding experimental measurements), respectively.

Inspecting the fitted parameters in Table I, one notices that the rescatterings inside the VP and VV systems proceed predominantly via the $S$-wave interactions. They can be described almost by one set of parameters, respectively, which is guaranteed by the heavy quark spin symmetry $[29,44]$. In addition, the LO LEC $\tilde{C}_{\mathrm{s}}$ for the charmed and bottom systems are consistent with each other within uncertainties, which is the reflection of heavy quark flavor symmetry $[29,45,46]$. The sensible difference of the NLO LEC $C_{\mathrm{s}}$ for the $D^{*} \bar{D}^{(*)}$ and $B^{*} \bar{B}^{(*)}$ systems encodes the heavy quark flavor symmetry breaking effect. The value of the cutoff $\Lambda$ also resides in the region $\left(\Lambda \ll \Lambda_{\chi}\right)$ where the $\chi$ EFT works healthily. The cutoff for the $B^{*} \bar{B}^{(*)}$ systems is larger than that of the $D^{*} \bar{D}^{(*)}$, since the interaction radius $(R \sim 1 / \Lambda)$ for the $B^{*} \bar{B}^{(*)}$ is shorter than that of the $D^{*} \bar{D}^{(*)}$.
It is well known that the bottom mesons are heavier than the charmed ones.

We also attempt to fit the data with the LO effective potentials solely (OPE plus the LO contact terms), but cannot reproduce the experimental line shapes well (purple dot-dashed lines in Fig. 3). Those bumps are caused by the sudden opening of the phase spaces together with the monotone decreasing behavior of the production amplitudes, but not by any genuine poles of the $T$ matrix in the second Riemann sheet. These signals become bound states with the LO interaction. Nevertheless, the parameters obtained with only the LO interaction are less reasonable, such as $\tilde{C}_{\mathrm{s}} \simeq-134.8 \mathrm{GeV}^{-2}$ and $\Lambda \simeq 1.37 \mathrm{GeV}$ for the $Z_{c}^{(\prime)}$ states (while $\tilde{C}_{\mathrm{s}} \simeq-29.3 \mathrm{GeV}^{-2}$ and $\Lambda \simeq 1.43 \mathrm{GeV}$ for the $Z_{b}^{(\prime)}$ states). Although there are no guidances to judge the

TABLE I. The fitted parameters for the $D^{*} \bar{D}^{(*)}$ and $B^{*} \bar{B}^{(*)}$ systems with the potentials up to the NLO, respectively. The LEC are in units of $10^{2}$. We define the masses and widths of the $Z_{Q}^{(\prime)}$ states from their pole positions $E=m-i \Gamma / 2$ (with $m$ the mass and $\Gamma$ the width). The masses and widths are given in units of $\mathrm{MeV}$. The correlation coefficients of the parameters in our fit are $\rho_{\tilde{C}_{\mathrm{s}} C_{\mathrm{s}}}=0.23$, $\rho_{\tilde{C}_{\mathrm{s}} C_{\mathrm{sd}}}=0.07, \rho_{\tilde{C}_{\mathrm{s}} \Lambda}=0.75, \rho_{C_{\mathrm{s}} C_{\mathrm{sd}}}=0.12, \rho_{C_{\mathrm{s}} \Lambda}=0.87$, and $\rho_{C_{\mathrm{sd}} \Lambda}=0.21$.

\begin{tabular}{lccccccc}
\hline \hline States & Thresholds & $\tilde{C}_{\mathrm{s}}\left[\mathrm{GeV}^{-2}\right]$ & $C_{\mathrm{s}}\left[\mathrm{GeV}^{-4}\right]$ & $C_{\mathrm{sd}}\left[\mathrm{GeV}^{-4}\right]$ & $\Lambda[\mathrm{GeV}]$ & {$[m, \Gamma]_{\text {pole }}$} & {$[m, \Gamma]_{\text {expt }}$.} \\
\hline$\frac{1}{\sqrt{2}}\left[D \bar{D}^{*}+D^{*} \bar{D}\right]$ & 3875.8 & $3.6_{-1.2}^{+1.2}$ & $-76.9_{-6.2}^{+6.2}$ & $1.1_{-5.8}^{+5.8}$ & $0.33_{-0.024}^{+0.024}$ & {$\left[3881.3_{-3.0}^{+3.0}, 12.4_{-5.0}^{+5.0}\right]$} & {$\left[3881.7_{-2.3}^{+2.3}, 26.6_{-3.0}^{+3.0}\right][14]$} \\
$D^{*} \bar{D}^{*}$ & 4017.1 & $4.0_{-1.6}^{+1.6}$ & $-78.1_{-8.7}^{+8.7}$ & $1.7_{-6.3}^{+6.3}$ & $0.34_{-0.031}^{+0.031}$ & {$\left[4026.5_{-4.5}^{+4.5}, 10.1_{-7.2}^{+7.2}\right]$} & {$\left[4025.5_{-5.6}^{+3.7}, 26.0_{-6.0}^{+6.0}\right][16]$} \\
$\frac{1}{\sqrt{2}}\left[B \bar{B}^{*}+B^{*} \bar{B}\right]$ & 10604.4 & $2.2_{-0.2}^{+0.2}$ & $-9.9_{-1.0}^{+1.0}$ & $3.6_{-4.7}^{+4.7}$ & $0.51_{-0.014}^{+0.014}$ & {$\left[10607.9_{-2.2}^{+2.2}, 10.9_{-3.0}^{+3.0}\right]$} & {$\left[10607.2_{-2.0}^{+2.0}, 18.4_{-2.4}^{+2.4}\right][17]$} \\
$B^{*} \bar{B}^{*}$ & 10649.4 & $2.2_{-0.3}^{+0.3}$ & $-9.9_{-1.2}^{+1.2}$ & $3.3_{-6.6}^{+6.6}$ & $0.51_{-0.015}^{+0.015}$ & {$\left[10652.8_{-2.7}^{+2.7}, 10.9_{-3.4}^{+3.4}\right]$} & {$\left[10652.2_{-1.5}^{+1.5}, 11.5_{-2.2}^{+2.2}\right][17]$} \\
\hline \hline
\end{tabular}


values of $\tilde{C}_{\mathrm{s}}$, the $\chi \mathrm{EFT}$ imposes strong constrains to the $\Lambda$, which has to be smaller than the typical hard scale, i.e., the $\rho$ meson mass $m_{\rho} \simeq 0.77 \mathrm{GeV}$. Therefore, we can conclude that either from the fitting quality or the rationality of parameters, the bound state explanations are not favored.

In addition, we notice the fitted $C_{\text {sd }}$ values in Table I are always compatible with zero. So we also try to re-fit the experimental data by turning off the $S-D$ mixing. We find the parameters $\tilde{C}_{s}, C_{s}$, and $\Lambda$ keep almost unchanged. On the one hand, this may indicate the influence of tensor force is not so important as in the low energy $N N$ interactions. On the other hand, the generally suppressed $D$-wave production vertex is not taken into account, this may partially weaken the influence of the $S-D$ mixing. The inelastic channels, e.g., $J / \psi \pi$ and $\Upsilon(n S) \pi$, can couple to the $Z_{c}^{(\prime)}$ and $Z_{b}^{(\prime)}$ states via an $S$ wave, respectively, so this couple channel effect may be more important than the $S-D$ mixing. As we explained above, the inelastic channel contributions cannot be accommodated in our framework, but an effective way with the couple channels could be utilized to phenomenologically analyze the inelastic channel contributions, which is given in the Appendix.

As elucidated above, the $Z_{Q}^{(\prime)}$ states can be well identified as the molecular resonances. In the resonance scenario, their decay behaviors can be explained qualitatively well. In contrast to the bound state, a resonance naturally dissolves to their components after interacting within finite time, which contributes to the dominant decay mode. The decays with the final states of a heavy quarkonium and a light meson $[Q \bar{q}]+[\bar{Q} q] \rightarrow[Q \bar{Q}]+[q \bar{q}]$ proceed with less probability, and they are induced by a much shorter range interaction (compared to $1 / \Lambda_{\chi}$ ). At the hadron level, these decays take place via exchanging a heavy meson $[Q \bar{q}]$, which is generally suppressed. This is why the partial widths from the inelastic channel contributions are much smaller than those of the elastic channels in experiments [13,18].

In summary, we systematically study the $D^{*} \bar{D}^{(*)}$ and $B^{*} \bar{B}^{(*)}$ effective potentials with the $\chi$ EFT up to the NLO to draw a clear picture of their interactions. With these potentials, we investigate the internal structures of the experimentally observed $Z_{c}^{(\prime)}$ and $Z_{b}^{(\prime)}$ states in recent years. The short-, mid- and long-range forces are all included to fit the invariant mass distributions. The experimental data are fitted very well with the effective potentials up to the NLO. The peaks in experiments arise from the poles in the second Riemann sheet, which indicate that the $Z_{c}^{(\prime)}$ and $Z_{b}^{(\prime)}$ states are resonances that are generated from the analogue of nuclear forces in heavy meson sectors. The heavy quark symmetry and its breaking effect are both reflected in the parameters. The fittings with the LO potentials give rise to the bound states, which is repudiated either by the abovethreshold masses or the validity of $\chi \mathrm{EFT}$. The decay behaviors of the $Z_{c}^{(\prime)}$ and $Z_{b}^{(\prime)}$ states can also be qualitatively interpreted in the resonance picture. In our study, the $Z_{Q}^{(\prime)}$ signals can be fully reproduced by the $\mathrm{VP}(\mathrm{V})$ rescatterings, where the initial states $\pi \mathrm{VP}(\mathrm{V})$ are assumed to be produced from pointlike sources. We do not need additional structures around the colliding energies.

Besides the $X Y Z$ states, more and more new states have been observed in experiments (such as the $P_{c}$ [47] and very recently reported $X_{0,1}$ states at $\mathrm{LHCb}[48,49]$ ), thus a model independent way is urgently called for to illuminate the nature of these new hadrons. The systematical generalization of the $\chi \mathrm{EFT}$ to the heavy meson systems is very successful in this work, which helps us to pin down the inner structures of the $Z_{c}^{(\prime)}$ and $Z_{b}^{(\prime)}$ states. This framework can also be applied to investigate whether the other nearthreshold states (e.g., $P_{c}$ and $X_{0,1}$ ) have the same origin, i.e., the dynamically generated resonances (bound states) from the analogue of nuclear forces in different sectors. This would undoubtedly deepen our understandings of the low energy behaviors of QCD.

\section{ACKNOLEDGMENTS}

This project is supported by the National Natural Science Foundation of China under Grant No. 11975033.

\section{APPENDIX: ESTIMATING THE INELASTIC CHANNEL CONTRIBUTIONS}

Although the inelastic channel contributions cannot be systematically included within the framework of $\chi$ EFT, which could be phenomenologically described in an effective way. Here, we take the $Z_{c}$ state as an example, and consider two channels, i.e., $D \bar{D}^{*}$ and $J / \psi \pi$ couplings. The effective potentials for these two channel couplings can be parametrized as

$$
V=\left[\begin{array}{cc}
0 & \mathcal{C} \\
\mathcal{C} & \tilde{\mathcal{C}}_{s}+\mathcal{C}_{s}\left(\boldsymbol{p}^{2}+\boldsymbol{p}^{\prime 2}\right)
\end{array}\right]
$$

where the $J / \psi$ and $\pi$ coupling strength is known to be tiny [50] and set to be 0 . The constant $\mathcal{C}$ represents the $D \bar{D}^{*}$ and $J / \psi \pi$ coupling, which can be regarded as the lowest order expansion in an EFT. The $\tilde{\mathcal{C}}_{s}+\mathcal{C}_{s}\left(\boldsymbol{p}^{2}+\boldsymbol{p}^{\prime 2}\right)$ depicts the $D$ and $\bar{D}^{*}$ interaction, which is analogous to Eq. (5).

The scattering $T$ matrix can be written as

$$
T=V+V G T,
$$

where the relativistic two-body propagator $G$ is defined as

$G(E+i \epsilon)=\int_{0}^{\Lambda} \frac{q^{2} d q}{(2 \pi)^{2}} \frac{\omega_{1}+\omega_{2}}{\omega_{1} \omega_{2}} \frac{1}{E^{2}-\left(\omega_{1}+\omega_{2}\right)^{2}+i \epsilon}$, 
with $\omega_{i}=\sqrt{\boldsymbol{q}^{2}+m_{i}^{2}}$ the energy of $i$ th particle of the two intermediate states. We use a sharp cutoff $\Lambda$ to regularize this loop integral. Considering the c.m.s. momentum of the $J / \psi \pi$ channel can reach up to $0.7 \mathrm{GeV}$ at the energy of $Z_{c}$, we choose $\Lambda=1.0$ and $0.5 \mathrm{GeV}$ for the $J / \psi \pi$ channel and $D \bar{D}^{*}$ channel, respectively.

With the on-shell approximation [51], the couple channel integral equation Eq. (A2) can be reduced to a set of algebraic equations, i.e.,

$$
T=(1-V G)^{-1} V
$$

Through analytical continuation of the Green's function $G$,

$$
G(E+i \epsilon) \rightarrow G(E+i \epsilon)+i \frac{q_{i}(E)}{4 \pi E},
$$

we search for the poles of the $T$ matrix in the second Riemann sheet by varying the values of the variables $\tilde{\mathcal{C}}_{s}, \mathcal{C}_{s}$, and $\mathcal{C}$ in the effective potentials. With the mass and width measured in Ref. [14] as inputs, we get

$$
\begin{aligned}
\tilde{\mathcal{C}}_{s} & =-0.16 \pm 0.02 \mathrm{GeV}^{-2}, \quad \mathcal{C}_{s}=-1.7 \pm 0.2 \mathrm{GeV}^{-4}, \\
\mathcal{C} & =0.03 \pm 0.01 \mathrm{GeV}^{-2},
\end{aligned}
$$

where the above values are in units of $10^{3}$. We notice the value of $\mathcal{C}$ is much smaller than that of the $\tilde{\mathcal{C}}_{s}$ and $\mathcal{C}_{s}$, this can qualitatively explain why the partial width $\Gamma\left[Z_{c} \rightarrow D \bar{D}^{*}\right]$ is much larger than the $\Gamma\left[Z_{c} \rightarrow J / \psi \pi\right]$ (note that the phase space of $Z_{c} \rightarrow D \bar{D}^{*}$ is much smaller than that of $\left.Z_{c} \rightarrow J / \psi \pi\right)$ [13]. Moreover, the above result indicates the inner structure of $Z_{c}$ is dominantly governed by the dynamics of $D \bar{D}^{*}$. This can also explain why we can give a rather good description of the experimental data although we do not consider the inelastic channel contributions.

The above analyses can also be applied to estimate the coupling strengths of $Z_{c}^{\prime}$ and $Z_{b}^{(\prime)}$ to the elastic and inelastic channels. The results should be very similar to the $Z_{c}$ state, since they are the twin partners under heavy quark limit, and the inelastic channels only contribute a small amount of partial widths [18].
[1] S. K. Choi et al. (Belle Collaboration), Observation of a Narrow Charmonium-Like State in Exclusive $B^{ \pm} \rightarrow$ $K^{ \pm} \pi^{+} \pi^{-} J / \psi$ Decays, Phys. Rev. Lett. 91, 262001 (2003).

[2] P. A. Zyla et al. (Particle Data Group), Review of particle physics, Prog. Theor. Exp. Phys. (2020) 083C01.

[3] H. X. Chen, W. Chen, X. Liu, and S. L. Zhu, The hiddencharm pentaquark and tetraquark states, Phys. Rep. 639, 1 (2016).

[4] F. K. Guo, C. Hanhart, U. G. Meißner, Q. Wang, Q. Zhao, and B. S. Zou, Hadronic molecules, Rev. Mod. Phys. 90, 015004 (2018).

[5] Y. R. Liu, H. X. Chen, W. Chen, X. Liu, and S. L. Zhu, Pentaquark and tetraquark states, Prog. Part. Nucl. Phys. 107, 237 (2019).

[6] R. F. Lebed, R. E. Mitchell, and E. S. Swanson, Heavyquark QCD exotica, Prog. Part. Nucl. Phys. 93, 143 (2017).

[7] A. Esposito, A. Pilloni, and A. D. Polosa, Multiquark resonances, Phys. Rep. 668, 1 (2017).

[8] N. Brambilla, S. Eidelman, C. Hanhart, A. Nefediev, C. P. Shen, C. E. Thomas, A. Vairo, and C.Z. Yuan, The $X Y Z$ states: Experimental and theoretical status and perspectives, Phys. Rep. 873, 1 (2020).

[9] M. Ablikim et al. (BESIII Collaboration), Observation of a Charged Charmoniumlike Structure in $e^{+} e^{-} \rightarrow$ $\pi^{+} \pi^{-} J / \psi$ at $\sqrt{s}=4.26 \mathrm{GeV}$, Phys. Rev. Lett. 110, 252001 (2013).

[10] M. Ablikim et al. (BESIII Collaboration), Observation of a Charged Charmoniumlike Structure $Z_{c}(4020)$ and Search for the $Z_{c}(3900)$ in $e^{+} e^{-} \rightarrow \pi^{+} \pi^{-} h_{c}$, Phys. Rev. Lett. 111, 242001 (2013).
[11] Z. Q. Liu et al. (Belle Collaboration), Study of $e^{+} e^{-} \rightarrow$ $\pi^{+} \pi^{-} J / \psi$ and Observation of a Charged Charmoniumlike State at Belle, Phys. Rev. Lett. 110, 252002 (2013).

[12] T. Xiao, S. Dobbs, A. Tomaradze, and K. K. Seth, Observation of the charged hadron $Z_{c}^{ \pm}(3900)$ and evidence for the neutral $Z_{c}^{0}(3900)$ in $e^{+} e^{-} \rightarrow \pi \pi J / \psi$ at $\sqrt{s}=4170 \mathrm{MeV}$, Phys. Lett. B 727, 366 (2013).

[13] M. Ablikim et al. (BESIII Collaboration), Observation of a Charged $\left(D \bar{D}^{*}\right)^{ \pm}$Mass Peak in $e^{+} e^{-} \rightarrow \pi D \bar{D}^{*}$ at $\sqrt{s}=$ 4.26 GeV, Phys. Rev. Lett. 112, 022001 (2014).

[14] M. Ablikim et al. (BESIII Collaboration), Confirmation of a charged charmoniumlike state $Z_{c}(3885)^{\mp}$ in $e^{+} e^{-} \rightarrow$ $\pi^{ \pm}\left(D \bar{D}^{*}\right)^{\mp}$ with double $D$ tag, Phys. Rev. D 92, 092006 (2015).

[15] M. Ablikim et al. (BESIII Collaboration), Observation of a Charged Charmoniumlike Structure in $e^{+} e^{-} \rightarrow\left(D^{*} \bar{D}^{*}\right)^{ \pm} \pi^{\mp}$ at $\sqrt{s}=4.26 \mathrm{GeV}$, Phys. Rev. Lett. 112, 132001 (2014).

[16] M. Ablikim et al. (BESIII Collaboration), Observation of a Neutral Charmoniumlike State $Z_{c}(4025)^{0}$ in $e^{+} e^{-} \rightarrow$ $\left(D^{*} \bar{D}^{*}\right)^{0} \pi^{0}$, Phys. Rev. Lett. 115, 182002 (2015).

[17] A. Bondar et al. (Belle Collaboration), Observation of Two Charged Bottomoniumlike Resonances in $\Upsilon(5 S)$ Decays, Phys. Rev. Lett. 108, 122001 (2012).

[18] A. Garmash et al. (Belle Collaboration), Observation of $Z_{b}(10610)$ and $Z_{b}(10650)$ Decaying to $B$ Mesons, Phys. Rev. Lett. 116, 212001 (2016).

[19] I. Adachi (Belle Collaboration), Observation of two charged bottomoniumlike resonances, arXiv:1105.4583.

[20] S. Weinberg, Nuclear forces from chiral Lagrangians, Phys. Lett. B 251, 288 (1990). 
[21] S. Weinberg, Effective chiral Lagrangians for nucleon-pion interactions and nuclear forces, Nucl. Phys. B363, 3 (1991).

[22] V. Bernard, N. Kaiser, and U. G. Meißner, Chiral dynamics in nucleons and nuclei, Int. J. Mod. Phys. E 04, 193 (1995).

[23] E. Epelbaum, H. W. Hammer, and U. G. Meißner, Modern theory of nuclear forces, Rev. Mod. Phys. 81, 1773 (2009).

[24] R. Machleidt and D. R. Entem, Chiral effective field theory and nuclear forces, Phys. Rep. 503, 1 (2011).

[25] U. G. Meißner, The long and winding road from chiral effective Lagrangians to nuclear structure, Phys. Scripta 91, 033005 (2016).

[26] H.-W. Hammer, S. König, and U. van Kolck, Nuclear effective field theory: Status and perspectives, Rev. Mod. Phys. 92, 025004 (2020).

[27] D. R. Entem, R. Machleidt, and Y. Nosyk, Nucleon-Nucleon scattering up to $\mathrm{N}^{5} \mathrm{LO}$ in chiral effective field theory, Front. Phys. 8, 57 (2020).

[28] M. B. Wise, Chiral perturbation theory for hadrons containing a heavy quark, Phys. Rev. D 45, R2188 (1992).

[29] A. V. Manohar and M. B. Wise, Heavy quark physics, Cambridge Monogr. Part. Phys., Nucl. Phys., Cosmol. 10, 1 (2000).

[30] B. Wang, L. Meng, and S. L. Zhu, $D^{(*)} N$ interaction and the structure of $\Sigma_{c}(2800)$ and $\Lambda_{c}(2940)$ in chiral effective field theory, Phys. Rev. D 101, 094035 (2020).

[31] B. Wang, L. Meng, and S. L. Zhu, Spectrum of the strange hidden charm molecular pentaquarks in chiral effective field theory, Phys. Rev. D 101, 034018 (2020).

[32] H. Ohki, H. Matsufuru, and T. Onogi, Determination of $B^{*} B \pi$ coupling in unquenched QCD, Phys. Rev. D 77, 094509 (2008).

[33] W. Detmold, C. J. D. Lin, and S. Meinel, Calculation of the heavy-hadron axial couplings $g_{1}, g_{2}$ and $g_{3}$ using lattice QCD, Phys. Rev. D 85, 114508 (2012).

[34] X. Liu, Y. R. Liu, W. Z. Deng, and S. L. Zhu, Is $Z^{+}(4430)$ a loosely bound molecular state?, Phys. Rev. D 77, 034003 (2008).

[35] V. B. Berestetsky, E. M. Lifshitz, and L. P. Pitaevsky, Quantum Electrodynamics (Butterworth-Heinemann Elsevier Ltd, Oxford, 1982).

[36] B. Wang, Z. W. Liu, and X. Liu, $\bar{B}^{(*)} \bar{B}^{(*)}$ interactions in chiral effective field theory, Phys. Rev. D 99, 036007 (2019).

[37] B. Wang, L. Meng, and S. L. Zhu, Hidden-charm and hidden-bottom molecular pentaquarks in chiral effective field theory, J. High Energy Phys. 11 (2019) 108.
[38] J. Golak et al., A new way to perform partial wave decompositions of few-nucleon forces, Eur. Phys. J. A 43, 241 (2010).

[39] E. Epelbaum, W. Glockle, and U. G. Meissner, The twonucleon system at next-to-next-to-next-to-leading order, Nucl. Phys. A747, 362 (2005).

[40] C. Hanhart, Y. S. Kalashnikova, P. Matuschek, R. V. Mizuk, A. V. Nefediev, and Q. Wang, Practical Parametrization for Line Shapes of Near-Threshold States, Phys. Rev. Lett. 115, 202001 (2015).

[41] M. Albaladejo, F. K. Guo, C. Hidalgo-Duque, and J. Nieves, $Z_{c}(3900)$ : What has been really seen?, Phys. Lett. B 755, 337 (2016).

[42] F.-K. Guo, C. Hanhart, Y. S. Kalashnikova, P. Matuschek, R. V. Mizuk, A. V. Nefediev, Q. Wang, and J.-L. Wynen, Interplay of quark and meson degrees of freedom in nearthreshold states: A practical parametrization for line shapes, Phys. Rev. D 93, 074031 (2016).

[43] Q. Wang, V. Baru, A. A. Filin, C. Hanhart, A. V. Nefediev, and J.-L. Wynen, Line shapes of the $Z_{b}(10610)$ and $Z_{b}(10650)$ in the elastic and inelastic channels revisited, Phys. Rev. D 98, 074023 (2018).

[44] J. Nieves and M.P. Valderrama, The heavy quark spin symmetry partners of the $X(3872)$, Phys. Rev. D 86, 056004 (2012).

[45] A. E. Bondar, A. Garmash, A. I. Milstein, R. Mizuk, and M. B. Voloshin, Heavy quark spin structure in $Z_{b}$ resonances, Phys. Rev. D 84, 054010 (2011).

[46] T. Mehen and J. W. Powell, Heavy quark symmetry predictions for weakly bound $B$-meson molecules, Phys. Rev. D 84, 114013 (2011).

[47] R. Aaij et al. (LHCb Collaboration), Observation of a Narrow Pentaquark State, $P_{c}(4312)^{+}$, and of Two-Peak Structure of the $P_{c}(4450)^{+}$, Phys. Rev. Lett. 122, 222001 (2019).

[48] R. Aaij et al. (LHCb Collaboration), A model-independent study of resonant structure in $B^{+} \rightarrow D^{+} D^{-} K^{+}$decays, arXiv:2009.00025.

[49] R. Aaij et al. (LHCb Collaboration), Amplitude analysis of the $B^{+} \rightarrow D^{+} D^{-} K^{+}$decay, arXiv:2009.00026.

[50] K. Yokokawa, S. Sasaki, T. Hatsuda, and A. Hayashigaki, First lattice study of low-energy charmonium-hadron interaction, Phys. Rev. D 74, 034504 (2006).

[51] J. A. Oller and E. Oset, Chiral symmetry amplitudes in the $S$ wave isoscalar and isovector channels and the $\sigma, f_{0}(980)$, $a_{0}(980)$ scalar mesons, Nucl. Phys. A620, 438 (1997). 